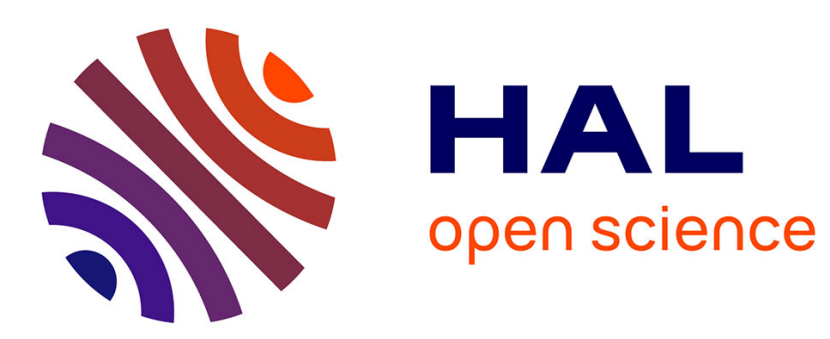

\title{
Méthode DDLTS utilisant une détection synchrone: application au GaAs implanté en oxygène
}

\author{
A. Le Bloa, Dang Tran Quan, P.N. Favennec
}

\section{To cite this version:}

A. Le Bloa, Dang Tran Quan, P.N. Favennec. Méthode DDLTS utilisant une détection synchrone: application au GaAs implanté en oxygène. Revue de Physique Appliquée, 1986, 21 (8), pp.489-499. 10.1051/rphysap:01986002108048900 . jpa-00245466

\section{HAL Id: jpa-00245466 https://hal.science/jpa-00245466}

Submitted on 1 Jan 1986

HAL is a multi-disciplinary open access archive for the deposit and dissemination of scientific research documents, whether they are published or not. The documents may come from teaching and research institutions in France or abroad, or from public or private research centers.
L'archive ouverte pluridisciplinaire HAL, est destinée au dépôt et à la diffusion de documents scientifiques de niveau recherche, publiés ou non, émanant des établissements d'enseignement et de recherche français ou étrangers, des laboratoires publics ou privés. 


\title{
Méthode DDLTS utilisant une détection synchrone : application au GaAs implanté en oxygène
}

\author{
A. Le Bloa, Dang Tran Quan et P. N. Favennec (*) \\ Groupe de Physique Cristalline, UA 040804 au C.N.R.S. Université de Rennes I, Campus de Beaulieu, \\ 35042 Rennes Cedex, France \\ $\left(^{*}\right)$ Groupement ICM/TOH, Centre National d'Etudes des Télécommunications, Lannion-B, \\ 22301 Lannion Cedex, France
}

(Reçu le 16 décembre 1985, révisé le 7 avril 1986, accepté le 25 avril 1986)

\begin{abstract}
Résumé. - On présente l'analyse détaillée d'une méthode DLTS à double corrélation utilisant une seule détection synchrone sélective et l'on montre l'intérêt qu'elle présente pour la détermination de la signature des centres profonds dans les semiconducteurs et du profil spatial de leur concentration. Cette méthode est appliquée à la caractérisation d'un échantillon de GaAs multi-implanté en oxygène. Une seule remontée en température est nécessaire pour faire la déconvolution des pics du spectre DDLTS relevé. On détermine ainsi la signature et l'énergie d'activation des défauts correspondants.
\end{abstract}

\begin{abstract}
A detailed analysis of a double correlation DLTS method using a selective lock-in amplifier is exposed. The interest of this method for determining the signature of the defects and their spatial depth profiles is explained. An application to oxygen multi-implanted GaAs sample is described. A single temperature scanning is needed to obtain the deconvolution of the three peaks of the observed spectrum. This operation permits to determine the " signature " and the activation energy of the corresponding defects.
\end{abstract}

\section{Introduction.}

Pour améliorer l'évaluation du profil de la concentration des défauts dans les semiconducteurs, Lefèvre et Schultz [1] ont mis au point une méthode DLTS à double corrélation (DDLTS). Johnson [2] a mis en œuvre cette méthode en maintenant constante la capacité de la diode pendant le régime transitoire au lieu de garder constante la polarisation appliquée; il a, d'autre part, utilisé cette méthode pour évaluer la densité des états d'interface des diodes MIS [3].

Les auteurs précédents utilisent la méthode DLTS de Lang [4] : ils enregistrent d'abord deux spectres DLTS puis en font la différence ce qui nécessite un double appareillage par rapport à la méthode classique de Lang. Comme des mesures de DLTS peuvent être effectuées en utilisant une détection synchrone sélective $[5,6]$, nous allons montrer que la méthode DDLTS peut aussi être réalisée avec une seule détection synchrone sélective, c'est-à-dire sans double appareillage.

\section{Théorie de la mesure.}

Nous présentons ici le principe de la méthode de mesure avant d'aborder les différents calculs néces- saires à l'évaluation de la signature d'un défaut et à la détermination de son profil spatial.

2.1 PRINCIPE DE LA MESURE. - Pendant chaque période $T_{0}$, nous superposons à la tension de polarisation inverse $V_{\mathbf{B}}$ de la diode deux impulsions de tension, d'amplitudes $V_{1}$ et $V_{2}\left(V_{1}>V_{2}\right)$, de même durée $t_{\mathrm{p}}$, décalées d'une demi-période $\left(T_{\mathrm{o}} / 2\right)$ (Fig. 1a). Désignons par :

- $N_{\mathrm{D}}, n(x), N_{t}(x)$ et $n_{t}(x, t)$ les concentrations à la distance $x$ respectivement des donneurs, des électrons libres, des défauts et des défauts occupés par un électron à l'instant $t$, l'origine des distances étant prise à la surface.

$-e_{n}$ et $C_{n}$ les taux d'émission et de capture thermique des électrons par les défauts.

$$
-f(x, t)=\frac{n_{t}(x, t) C_{n}}{e_{n}+n_{t}(x, t) C_{n}}=\frac{n_{t}(x, t)}{N_{t}(x)} \text { la fonction }
$$

de remplissage des défauts à la distance $x$ et à l'instant $t$.

$-x_{1}$ et $x_{2}$ les positions (Fig. 2) où $f(x, t)=1 / 2$ à la fin respectivement de la première impulsion $\left(t=-t_{\mathrm{d}}\right)$ et de la deuxième impulsion de tension $\left(t=T_{0} / 2-t_{\mathrm{d}}\right)$.

$-x_{\mathrm{D}_{1}}(t)$ et $x_{\mathrm{D}_{2}}(t)$ les positions (Fig. 2) où $f(x, t)=1 / 2$ dans l'intervalle de temps entre deux impulsions de 


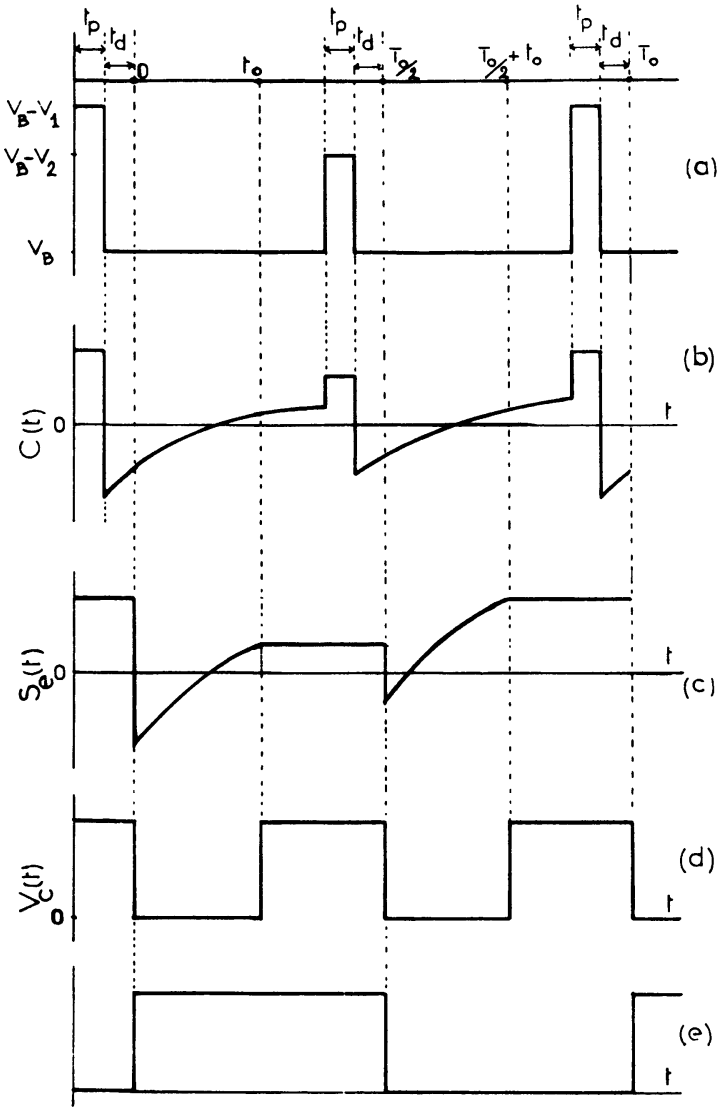

Fig. 1. - Chronogramme des signaux : (a) signal de polarisation de la diode; (b) signal de sortie du capacimètre; (c) signal de sortie de l'échantillonneur-bloqueur ; (d) signal de commande de l'échantillonneur-bloqueur; (e) signal de référence de la détection synchrone.

[Signal chronogram : (a) diode bias ; (b) output signal of the capacimeter; (c) output signal of the sample-hold module; (d) control signal of the sample-hold module; (e) reference signal of the lock-in amplifier.]

tension respectivement pendant la première $\left(-t_{\mathrm{d}}<t<T_{0} / 2-t_{\mathrm{d}}-t_{\mathrm{p}}\right)$ et la deuxième demipériode $\left(T_{0} / 2-t_{\mathrm{d}}<t<T_{0}-t_{\mathrm{d}}-t_{\mathrm{p}}\right)$.

- $W_{1}(t)$ et $W_{2}(t)$ les largeurs de la charge d'espace de la diode (Fig. 2) dans l'intervalle de temps entre deux impulsions de tension respectivement pendant la première $\left(-t_{\mathrm{d}}<t<T_{0} / 2-t_{\mathrm{d}}-t_{\mathrm{p}}\right)$ et la deuxième demi-période $\left(T_{0} / 2-t_{\mathrm{d}}<t<T_{0}-t_{\mathrm{p}}-t_{\mathrm{d}}\right)$.

Lorsque la durée $t_{\mathrm{p}}$ de l'impulsion de tension de polarisation est plus grande que cinq fois la constante de temps de capture des porteurs dans la région électriquement neutre du semiconducteur, Pons $[7,8]$ a montré que l'approximation suivante pour la fonction de remplissage $f\left(x,-t_{\mathrm{d}}\right)$ ou $f\left(x, T_{0} / 2-t_{\mathrm{d}}\right)$ est satisfaisante :

$f\left(x,-t_{\mathrm{d}}\right)\left(\right.$ ou $\left.f\left(x, T_{0} / 2-t_{\mathrm{d}}\right)\right)\left|\begin{array}{l}0 \text { si } x<x_{1}\left(\text { ou } x_{2}\right) \\ 1 \text { si } x>x_{1}\left(\text { ou } x_{2}\right)\end{array}\right|$

Les défauts remplis d'électrons pendant les impul-

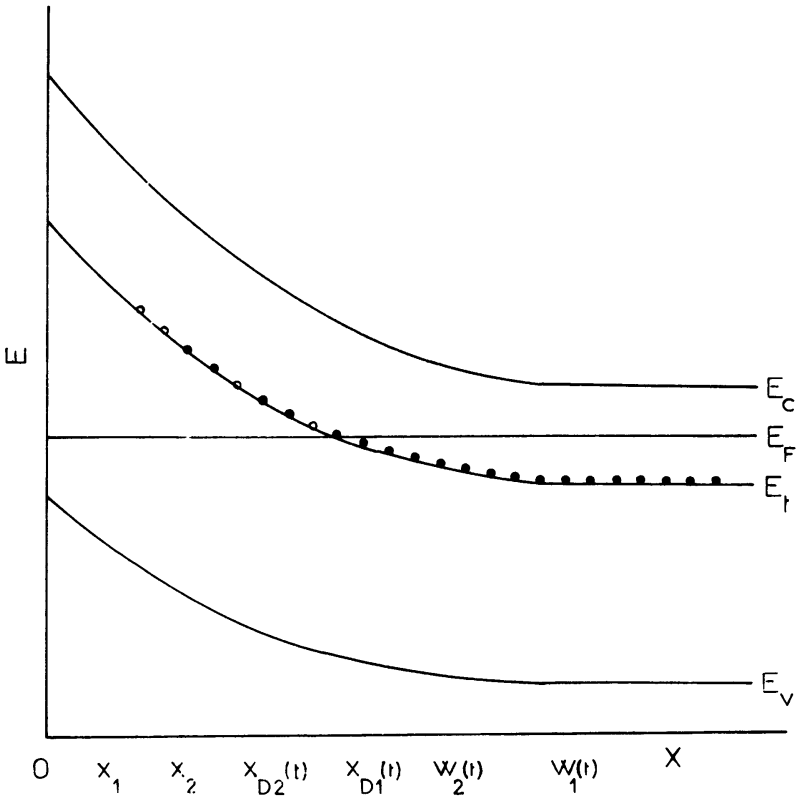

Fig. 2. - Diagramme des bandes et niveau d'énergie $E_{t}$ d'un défaut discret dans la charge d'espace d'une diode Schottky. $x_{1}$ et $x_{2}$ sont les positions où la fonction de remplissage $f(x, t)=1 / 2$ à la fin respectivement de la première $\left(t_{0}=-t_{\mathrm{d}}\right)$ et de la deuxième $\left(t=T_{0} / 2-t_{\mathrm{d}}\right)$ impulsion de tension; $x_{\mathrm{D}_{1}}(t)$ et $x_{\mathrm{D}_{2}}(t)$ sont les positions où $f(x, t)=1 / 2$ pour $-t_{\mathrm{d}}<t<T_{0} / 2-t_{\mathrm{p}}-t_{\mathrm{d}}$ et $T_{0} / 2-t_{\mathrm{d}}<t<T_{0}-$ $-t_{\mathrm{p}}-t_{\mathrm{d}} ; W_{1}(t)$ et $W_{2}(t)$ sont les largeurs de la charge d'espace de la diode dans les intervalles de temps entre les impulsions de tension respectivement pendant la première et pendant la deuxième demi-période.

[Schematic energy-band diagram for a Schottky barrier with a single discrete defect level at an energy $E_{t}$ in the semiconductor band gap. The distances $x_{1}$ and $x_{2}$ are the depths where $f(x, t)=1 / 2$ at the end of the first $\left(t=-t_{\mathrm{d}}\right)$ and at the end of the second $\left(t=T_{0} / 2-t_{\mathrm{d}}\right)$ refilling pulse respectively; $x_{D_{1}}(t)$ and $x_{D_{2}}(t)$ are the depths where $f(x, t)=1 / 2$ for $-t_{\mathrm{d}}<t<T_{0} / 2-t_{\mathrm{p}}-t_{\mathrm{d}}$ and for $T_{0} / 2-t_{\mathrm{d}}<t<$ $T_{0}-t_{\mathrm{p}}-t_{\mathrm{d}} ; W_{1}(t)$ and $W_{2}(t)$ are the width of spacecharge regions for the first and the second half-period respectively.]

sions de tension de polarisation jusqu'aux distances $x>x_{1}$ (ou $x_{2}$ ) (l'origine des distances est prise à la surface) vont, dans l'intervalle de temps entre les impulsions de tension, émettre leurs électrons vers la bande de conduction; cette émission produit une évolution exponentielle de la capacité de la diode (Fig. 1b); un échantillonneur-bloqueur permet de n'appliquer ce signal de sortie du capacimètre à l'entrée de la détection synchrone que pendant une durée $t_{0}$ (Fig. 1c) avec un retard $t_{\mathrm{d}}=1,6 \mathrm{~ms}$ [6] par rapport à la fin de l'impulsion de tension de polarisation : on rend ainsi négligeable l'influence de la réponse transitoire du capacimètre sur les mesures. Le signal de commande de l'échantillonneur-bloqueur (Fig. 1d) a une fréquence double de celle du signal de référence de la détection synchrone (Fig. 1e). 
2.2 EXPRESSION DE LA VARIATION DE CAPACITÉ D'UNE DIODE PENDANT LA POLARISATION INVERSE. - Nous exprimerons d'abord la différence de potentiel aux bornes de la diode en fonction des charges électriques présentes dans sa charge d'espace : $V$ est la somme de la tension appliquée à la diode et de la hauteur de la barrière de potentiel à la jonction hors polarisation.

Dans les intervalles de temps entre deux impulsions de tension, l'émission des porteurs par les défauts est prépondérante par rapport à leur capture entre $x_{1}$ et $x_{D_{1}}(t)$ ou entre $x_{2}$ et $x_{D_{2}}(t)$.

On déduit alors de l'équation de continuité :

- pour $-t_{\mathrm{d}}<t<T_{\mathrm{o}} / 2-\left(t_{\mathrm{d}}+t_{\mathrm{p}}\right)$

$$
n_{t}(x, t)=N_{t}(x) \exp \left[-e_{n}\left(t+t_{\mathrm{d}}\right)\right]
$$

- pour $T_{0} / 2-t_{\mathrm{d}}<t<T_{0}-\left(t_{\mathrm{d}}+t_{\mathrm{p}}\right)$

$$
n_{t}^{\prime}(x, t)=N_{t}(x) \exp \left[-e_{n}\left(t+t_{\mathrm{d}}-T_{0} / 2\right)\right] .
$$

Nous considérons qu'aux instants initiaux $t=-t_{\mathrm{d}}$ et $t=T_{0} / 2-t_{\mathrm{d}}$ les défauts sont tous occupés par des électrons (l'origine des temps est retardée de $t_{\mathrm{d}}$ par rapport à la fin de l'impulsion de tension Fig. 1a).

Le tableau I donne, pour les deux demi-périodes et en fonction de la distance au contact Schottky, la densité volumique $\rho(x, t)$ des charges électriques contenues dans la charge d'espace de la diode.

L'expression de la tension de polarisation $V$ déduite de l'équation de Poisson s'écrit [2] :

$$
V=\int_{0}^{W(t)} \frac{x \rho(x, t)}{\varepsilon} \mathrm{d} x
$$

$\varepsilon$ est la constante diélectrique du semiconducteur

$$
\begin{aligned}
& W(t)=W_{1}(t) \quad \text { dans l'intervalle }-t_{\mathrm{d}}<t<T_{0} / 2-t_{\mathrm{d}}-t_{\mathrm{p}} \\
& W(t)=W_{2}(t) \text { dans l'intervalle } T_{0} / 2-t_{\mathrm{d}}<t<T_{0}-t_{\mathrm{d}}-t_{\mathrm{p}} .
\end{aligned}
$$

En utilisant les expressions de $\rho(x, t)$ du tableau I, développons (3) pour les deux domaines de $t$ :

- pour $-t_{\mathrm{d}}<t<T_{\mathrm{o}} / 2-\left(t_{\mathrm{d}}+t_{\mathrm{p}}\right)$

$$
\begin{aligned}
& \frac{\varepsilon V}{q}=\int_{0}^{x_{1}} x\left(N_{\mathrm{D}}+N_{\mathrm{t}}(x)\right) \mathrm{d} x+\int_{x_{1}}^{x_{\mathrm{D}_{1}}(t)} x\left[N_{\mathrm{D}}+N_{\mathrm{t}}(x)-n_{\mathrm{t}}(x, t)\right] \mathrm{d} x+\int_{x_{\mathbf{D}_{1}}(t)}^{W_{1}(t)} x N_{\mathrm{D}} \mathrm{d} x \\
&-\operatorname{pour} \frac{T_{0}}{2}-t_{\mathrm{d}}<t<T_{0}-\left(t_{\mathrm{p}}+t_{\mathrm{d}}\right) \\
& \frac{\varepsilon V}{q}=\int_{0}^{x_{1}} x\left[N_{\mathrm{D}}+N_{\mathrm{t}}(x)\right] \mathrm{d} x+\int_{x_{1}}^{x_{2}} x\left[N_{\mathrm{D}}+N_{\mathrm{t}}(x)-n_{\mathrm{t}}(x, t)\right] \mathrm{d} x+ \\
&+\int_{x_{2}}^{x_{\mathbf{D}_{2}}(t)} x\left[N_{\mathrm{D}}+N_{\mathrm{t}}(x)-n_{\mathrm{t}}^{\prime}(x, t)\right] \mathrm{d} x+\int_{x_{\mathrm{D}_{2}}(t)}^{W_{2}(t)} x N_{\mathrm{D}} \mathrm{d} x
\end{aligned}
$$

où $q$ est la charge élémentaire.

Les charges électriques positives contenues dans les défauts (donneurs) augmentent avec le temps. Par ailleurs, les tensions de polarisation sont maintenues constantes; on déduit alors de l'équation (3) que :

$$
W_{1}(t)<W_{1}\left(-t_{\mathrm{d}}\right) \quad \text { et } \quad W_{2}(t)<W_{2}\left(-t_{\mathrm{d}}+\frac{T_{0}}{2}\right) \quad \text { et } \quad W_{1}(t)>W_{2}(t)
$$

Tableau I. - Valeurs de la densité volumique des charges électriques $\rho(x, t)$ dans la charge d'espace de la diode, en fonction de la distance $x$ à la surface et dans les intervalles de temps entre deux impulsions de tension; les défauts sont supposés être des donneurs.

[Values of the electric charge density $\rho(x, t)$ versus distance $x$ in the space-charge region of the diode between two filling pulses. (Case of donnor defects).]

\begin{tabular}{|c|c|c|c|c|c|}
\hline$x$ & 0 & \multicolumn{3}{|c|}{$x_{2} x_{\mathrm{D}_{1}}(t)$} & \multicolumn{3}{|c|}{$W_{1}(t) x_{\mathrm{D}_{2}}(t) W_{2}(t)$} \\
\hline$-t_{\mathrm{d}}<t<\frac{T_{0}}{2}-\left(t_{\mathrm{d}}+t_{\mathrm{p}}\right)$ & $N_{\mathrm{D}}+N_{\mathrm{t}}(x)$ & $N_{\mathrm{D}}+N_{\mathrm{t}}(x)-n_{\mathrm{t}}(x, t)$ & $N_{\mathrm{D}}$ & 0 & 0 \\
\hline$\frac{T_{0}}{2}-t_{\mathrm{d}}<t<T_{0}-\left(t_{\mathrm{d}}+t_{\mathrm{p}}\right)$ & $N_{\mathrm{D}}+N_{\mathrm{t}}(x)$ & $N_{\mathrm{D}}+N_{\mathrm{t}}(x)-n_{\mathrm{t}}(x, t)$ & $N_{\mathrm{D}}+N_{\mathrm{t}}(x)-n_{\mathrm{t}}^{\prime}(x, t)$ & $N_{\mathrm{D}}$ & 0 \\
\hline
\end{tabular}


ce qui implique : $x_{\mathrm{D}_{1}}(t)<x_{\mathrm{D}_{1}}\left(-t_{\mathrm{d}}\right)<W_{1}(t)<W_{1}\left(-t_{\mathrm{d}}\right)$ pour la première période (Fig. 2) et

$$
x_{\mathrm{D}_{2}}(t)<x_{\mathrm{D}_{2}}\left(-t_{\mathrm{d}}+\frac{T_{0}}{2}\right)<W_{2}(t)<W_{2}\left(-t_{\mathrm{d}}+\frac{T_{0}}{2}\right)
$$

pour la deuxième demi-période.

(On notera qu'à la fin des impulsions de tension on a $: t=-t_{\mathrm{d}}$ et $t=-t_{\mathrm{d}}+T_{0} / 2$.)

En supposant $e_{n}$ indépendant de $x$ et en prenant une valeur de $t$ telle que : $-t_{\mathrm{d}}<t<T_{0} / 2-\left(t_{\mathrm{p}}+t_{\mathrm{d}}\right)$, l'équation (4) permet d'écrire :

$$
\left[1-\exp \left(-e_{n}\left(t+t_{\mathrm{d}}\right)\right)\right] \int_{x_{1}}^{x_{\mathrm{D}_{1}}(t)} x N_{\mathrm{t}}(x) \mathrm{d} x=\frac{\bar{N}_{\mathrm{D}}}{2}\left[W_{1}^{2}\left(-t_{\mathrm{d}}\right)-W_{1}^{2}(t)\right]
$$

$\bar{N}_{\mathrm{D}}$ est la valeur moyenne de $N_{\mathrm{D}}$ dans l'intervalle $\left[W_{1}(t), W_{1}\left(-t_{\mathrm{d}}\right)\right]$.

Posons $C_{1}(t)=\varepsilon S / W_{1}(t)\left(S\right.$ : aire de la diode) et $\Delta C_{1}(t)=C_{1}(t)-C_{1}\left(-t_{\mathrm{d}}\right)$. Lorsque $N_{t}(x) \ll \bar{N}_{\mathrm{D}}$ nous avons $x_{\mathrm{D}_{1}}(t) \approx x_{\mathrm{D}_{1}}\left(-t_{\mathrm{d}}\right), \bar{N}_{\mathrm{D}}=N_{\mathrm{D}}\left(W_{1}\left(-t_{\mathrm{d}}\right)\right)$ et $C_{1}(t) \approx C_{1}\left(-t_{\mathrm{d}}\right)=C_{0}$; dans ce cas nous déduisons de l'équation (6) :

$$
\Delta C_{1}(t) \approx \frac{C_{0}^{3}}{\bar{N}_{\mathrm{D}} \varepsilon^{2} S^{2}}\left[1-\exp \left(-e_{n}\left(t_{\mathrm{d}}+t\right)\right)\right] \int_{x_{1}}^{x_{\mathrm{D}_{1}}\left(-t_{\mathrm{d}}\right)} x N_{\mathrm{t}}(x) \mathrm{d} x
$$

Dans l'intervalle de temps $] \frac{T_{0}}{2}-t_{\mathrm{d}}, T_{0}-t_{\mathrm{d}}-t_{\mathrm{p}}[$ l'équation (5) permet d'écrire une relation semblable à la relation (6) :

$$
\begin{aligned}
{\left[1-\exp \left(-e_{n}\left(t+t_{\mathrm{d}}-T_{0} / 2\right)\right)\right]\left[\exp \left(-e_{n} T_{0} / 2\right) \int_{x_{1}}^{x_{2}} x N_{t}(x) \mathrm{d} x\right.} & \left.+\int_{x_{2}}^{x_{\mathrm{D}_{2}}(t)} x N_{t}(x) \mathrm{d} x\right]= \\
& =\frac{\bar{N}_{\mathrm{D}}}{2}\left[W_{2}^{2}\left(-t_{\mathrm{d}}+T_{0} / 2\right)-W_{2}^{2}(t)\right] .
\end{aligned}
$$

La première et la deuxième intégrale représentent la contribution, à la variation de la charge électrique de la charge d'espace, des défauts remplis d'électrons à la fin respectivement de la première et de la deuxième impulsion de tension de polarisation (domaine $] x_{1}, x_{2}[$ et $] x_{2}, x_{\mathbf{D}_{2}}(t)[$ ).

Comme pour la première demi-période, considérons que $N_{t}(x) \ll \bar{N}_{\mathrm{D}}$ et posons :

$$
C_{2}(t)=\varepsilon S / W_{2}(t), \quad \Delta C_{2}(t)=C_{2}(t)-C_{2}\left(-t_{\mathrm{d}}+T_{0} / 2\right) \quad \text { et } \quad C_{2}\left(-t_{\mathrm{d}}+T_{0} / 2\right) \approx C_{0} ;
$$

on déduit alors de l'équation (8) une relation semblable à l'équation (7) :

$$
\Delta C_{2}(t) \approx \frac{C_{0}^{3}}{\bar{N}_{\mathrm{D}} \varepsilon^{2} S^{2}}\left[1-\exp \left(-e_{n}\left(t+t_{\mathrm{d}}-T_{0} / 2\right)\right)\right]\left[\mathrm{e}^{-\frac{\mathrm{e}_{n} T_{0}}{2}} \int_{x_{1}}^{x_{2}} x N_{\mathrm{t}}(x) \mathrm{d} x+\int_{x_{2}}^{x_{\mathrm{D}}\left(T_{0} / 2-t_{\mathrm{d}}\right)} x N_{\mathrm{t}}(x) \mathrm{d} x\right] .
$$

Dans la suite nous considérons que $N_{\mathrm{t}}(x) \ll \bar{N}_{\mathrm{D}}$ et nous avons alors $\bar{N}_{\mathrm{D}} \approx N_{\mathrm{D}}(W)$ avec $W \approx W_{1}(t) \approx W_{2}(t)$.

2.3 CALCUL DE L'AMPLITUde DES SIGNAUX DE SORTIE DE LA DÉTECTION SYNCHRONE. - $-\Delta C_{1}(t)$ et $\Delta C_{2}(t)$ représentent respectivement les variations de la capacité de la diode après la première et la deuxième impulsion de tension; ces variations sont appliquées à l'entrée de l'échantillonneur-bloqueur dont le signal de sortie, (lui-même appliqué à l'entrée de la détection synchrone), est donné, selon la valeur de $t$, par l'une des expressions suivantes :

$$
f(t)=\mid \begin{array}{ll}
\Delta C_{1}(t) & \text { pour } 0<t<t_{0} \\
\Delta C_{1}\left(t_{0}\right) & \text { pour } t_{0}<t<T_{0} / 2 \\
\Delta C_{2}(t) & \text { pour } \frac{T_{0}}{2}<t<\left(t_{0}+T_{0} / 2\right) \\
\Delta C_{2}\left(t_{0}+T_{0} / 2\right) & \text { pour } t_{0}+T_{0} / 2<t<T_{0}
\end{array}
$$


Les sorties de la détection synchrone fournissent les valeurs des coefficients de Fourier $\left(a_{1}\right.$ et $\left.b_{1}\right)$ de la fondamentale de $f(t)$ :

$$
\begin{aligned}
& a_{1}=\frac{2}{T_{0}} \int_{0}^{T_{0}} f(t) \cos \omega t \mathrm{~d} t \\
& b_{1}=\frac{2}{T_{0}} \int_{0}^{T_{0}} f(t) \sin \omega t \mathrm{~d} t .
\end{aligned}
$$

Ces coefficients sont fonction de la durée $t_{0}$ (Fig. 1) de l'échantillonnage; la limite supérieure de $t_{0}$ est $\left[\left(T_{0} / 2\right)-\left(t_{\mathrm{p}}+t_{\mathrm{d}}\right)\right] ; t_{0}$ dépend donc de la durée $t_{\mathrm{p}}$ de l'impulsion de tension de polarisation; c'est pour cette raison que nous avons choisi seulement deux valeurs remarquables de $t_{0}: T_{0} / 4$ et $3 T_{0} / 8$ pour calculer les coefficients $a_{1}, b_{1}$; les valeurs obtenues en fonction de $x=\omega / e_{n}$ sont données dans le tableau II où nous posons :

$$
B=\frac{C_{0}^{3}}{N_{\mathrm{D}}(W) \varepsilon^{2} S^{2}} \int_{x_{1}}^{x_{2}} x N_{t}(x) \mathrm{d} x .
$$

Les signaux de sortie de la détection synchrone ne dépendent donc que des défauts présents dans $] x_{1}, x_{2}[$.

Les variations de chacun des coefficients $a_{1}, b_{1}$ sont représentées par une courbe ayant un seul maximum. Les maxima $a_{1, \mathrm{~m}}, b_{1, \mathrm{~m}}$ de ces coefficients ainsi que les valeurs $x_{\mathrm{m}}$ de $x$ correspondantes sont, dans le cas $B=1$, représentés en fonction de la fréquence sur la figure 3 ; nous verrons par la suite que $a_{1, \mathrm{~m}}, b_{1, \mathrm{~m}}$ et $x_{\mathrm{m}}$ sont nécessaires pour déterminer les taux d'émission $e_{n}$ en fonction de la température. Les coefficients de Fourier ne dépendent de $\omega$ que par le terme $\exp \left(-\omega t_{\mathrm{d}} / x\right)$, leurs maxima $a_{1, \mathrm{~m}}, b_{1, \mathrm{~m}}$ diminuent lorsque la fréquence de mesure croît; comme pour la méthode DLTS [6] cette diminution des amplitudes

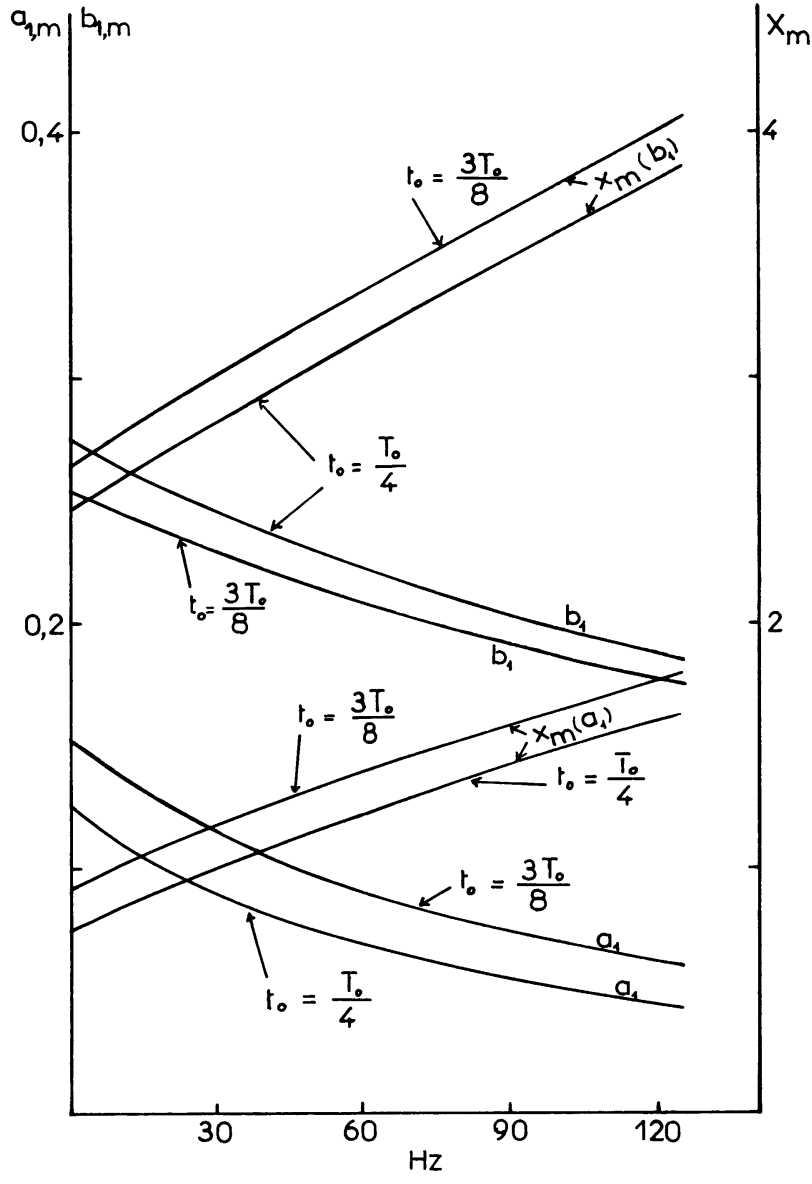

Fig. 3. - Variations en fonction de la fréquence, aux valeurs de $t_{0}=T_{0} / 4$ et $t_{0}=3 T_{0} / 8$, de : - l'amplitude maximale de $a_{1}$ et de $b_{1}(B=1) ;-x_{\mathrm{m}}$, valeur de $x=\omega / e_{n}$ correspondant à ces maxima.

[Variations versus frequency for $t_{0}=T_{0} / 4$ and $t_{0}=3 T_{0} / 8$ of : - maximum amplitude of $a_{1}$ and $b_{1}(B=1) ;-x_{\mathrm{m}}$, value of $x=\omega / e_{n}$ corresponding to their maxima.]

Tableau II. - Expressions des signaux de sortie d'une détection synchrone pour $t_{0}=\frac{T_{0}}{4}$ et $t_{0}=\frac{3 T_{0}}{8}$ en posant $x=\omega / e_{n}$.

[Output signals of the lock-in amplifier for $t_{0}=T_{0} / 4$ and $t_{0}=3 T_{0} / 8$ with $x=\omega / e_{n}$.]

\begin{tabular}{l|l|l}
$t_{0}=\frac{T_{0}}{4}$ & $a_{1}$ & $B \frac{\exp \left(-\omega t_{\mathrm{d}} / x\right)[1-\exp (-\pi / x)]}{\pi\left(1+x^{2}\right)}[x-\exp (-\pi / 2 x)]$ \\
\cline { 2 - 4 } & $b_{1}$ & $B \frac{\exp \left(-\omega t_{\mathrm{d}} / x\right)[1-\exp (-\pi / x)]}{\pi\left(1+x^{2}\right)}\left[x^{2}+\exp (-\pi / 2 x)\left(x^{2}+1-x\right)\right]$ \\
\hline \multirow{2}{*}{$t_{0}=\frac{3 T_{0}}{8}$} & $a_{1}$ & $B \frac{\exp \left(-\omega t_{\mathrm{d}} / x\right)[1-\exp (-\pi / x)]}{\pi\left(1+x^{2}\right)}[x-(\sqrt{2} / 2)(1-x) \exp (-3 \pi / 4 x)]$ \\
\cline { 2 - 3 } & $b_{1}$ & $B \frac{\exp \left(-\omega t_{\mathrm{d}} / x\right)[1-\exp (-\pi / x)]}{\pi\left(1+x^{2}\right)}\left[x^{2}+\exp (-3 \pi / 4 x)\left(x^{2}+1-\frac{\sqrt{2}}{2}(1+x)\right)\right]$
\end{tabular}


maximales avec l'augmentation de la fréquence est accompagnée d'une amélioration de la sélectivité. Les variations de toutes les fonctions du tableau II en fonction de $x$, c'est-à-dire de $e_{n}$ ou de $T$ (température), sont représentées par des courbes en forme de pic; au cours d'une remontée de température un spectre DDLTS présente très souvent plusieurs pics; chaque pic correspond à un défaut.

Le tableau III donne les variations asymptotiques des trois coefficients aux grandes valeurs de $\tau$ ainsi que l'ordre du filtre que constitue la détection synchrone, dans la nomenclature de Crowell et al. [9].

La détection synchrone mesurant $a_{1}$ est un filtre d'ordre 2 ; la sélectivité est donc meilleure pour $a_{1}$ que pour $b_{1}$ (ordre 1).

2.4 Signature D'UN DÉFAUT. - L'expression du taux d'émission d'un électron par un défaut est donnée par [10] :

$$
e_{n}=\gamma \sigma_{\infty} T^{2} \exp \left(-E_{\mathrm{a}} / k T\right)
$$

où $\gamma$ est un facteur dépendant du semiconducteur, $T$ la température thermodynamique, $E_{\mathrm{a}}$ l'énergie d'activation du taux d'émission, $k$ la constante de Boltzmann et $\sigma_{\infty}$ la section efficace de capture apparente d'un électron.

Pour évaluer le taux d'émission $e_{n}$ d'un défaut en fonction de la température, on recherche pour chaque valeur choisie de $S(T)$ du pic correspondant, la solution $x=\omega / e_{n}$ de l'équation :

$$
g(x)=\left(a_{1} / a_{1, \mathrm{~m}}-S(T) / S_{\mathrm{m}}\right)
$$

avec :

$a_{1}$ l'expression issue du tableau II avec $B=1$.

$a_{1, \mathrm{~m}}$ sa valeur maximale à la fréquence et au mode DDLTS utilisé $\left(t_{0}=T_{0} / 4\right.$ ou $\left.t_{0}=3 T_{0} / 8\right)$.

$S_{\mathrm{m}}$ la valeur de $S(T)$ au maximum du pic.

La normalisation de $a_{1}$ et de $S(T)$ à leur maximum réduit le nombre d'inconnues de deux $(B, x)$ à une $(x)$. Une résolution numérique (méthode de Newton) de cette équation permet d'obtenir $x$ et par conséquent

Tableau III. - Variations asymptotiques des coefficients $a_{1}$ et $b_{1}$ aux valeurs élevées de la constante de temps $\tau$ et ordre du filtre dans la nomenclature de Crowell et al. [9].

[Asymptotic variations of $a_{1}$ and $b_{1}$ for high values of $\tau$ and filter order after Crowell et al. [9] classification.]

\begin{tabular}{c|c|c} 
Coefficients & $\begin{array}{c}\text { Variations } \\
\text { asymptotiques } \\
\text { pour } \tau \text { grand }\end{array}$ & Ordre du filtre \\
\hline$a_{1}$ & $\tau^{-2}$ & 2 \\
$b_{1}$ & $\tau^{-1}$ & 1
\end{tabular}

$e_{n}$ à la température de chaque point de mesure. Une procédure semblable, appliquée au signal $b_{1}$ du même défaut, permet d'obtenir de nouvelles valeurs de $e_{n}$. On détermine ainsi la (ou les) signature du (ou des) défaut à partir du spectre DDLTS relevé au cours d'une seule remontée de température. Une régression linéaire de $\log \left(T^{2} / e_{n}\right)=f(1 / T)$ permet d'évaluer $\sigma_{\infty}$, $E_{\mathrm{a}}$ (à partir de la pente $E_{\mathrm{a}} / k$ ) et $s$, écart type qui permet d'estimer l'erreur.

Quand deux pics sont partiellement superposés, il est encore possible d'obtenir la signature de chacun des centres correspondants par la procédure précédente si le chevauchement n'est pas trop important mais les signatures évaluées sont alors moins précises.

2.5 VARIATIONS DU SIGNAL DDLTS EN FONCTION DE LA DIFFÉRENCE D'AMPLITUDE ENTRE LES DEUX IMPULSIONS DE TENSION. - Pons [7,8] a effectué le calcul de l'amplitude du transitoire de capacité $\Delta C_{\mathrm{m}}\left(t_{\mathrm{p}}\right)$ d'une diode en fonction de l'amplitude $\Delta V$ et de la durée $t_{\mathrm{p}}$ de l'impulsion de tension de polarisation pour des concentrations constantes des donneurs $N_{\mathrm{D}}$ et des défauts $N_{t}(x)$ du substrat de la diode et pour $N_{t}(x) \ll N_{\mathrm{D}}$. Comme l'amplitude du signal DLTS est proportionnelle à $\Delta C_{\mathrm{m}}\left(t_{\mathrm{p}}\right)$ il a vérifié [11] qu'au-dessus d'un seuil de tension dépendant de $t_{\mathrm{p}}$, la variation en fonction de $\Delta V$ de l'amplitude du signal DLTS, au maximum d'un pic, est quasi-linéaire dans un domaine assez grand de $\Delta V$.

Le signal DDLTS est, lui, proportionnel à la différence $\left[\Delta C_{\mathrm{m}, 1}\left(t_{\mathrm{p}}\right)-\Delta C_{\mathrm{m}, 2}\left(t_{\mathrm{p}}\right)\right]$ où $\Delta C_{\mathrm{m}, 1}\left(t_{\mathrm{p}}\right)$ et $\Delta C_{\mathrm{m}, 2}\left(t_{\mathrm{p}}\right)$ sont les amplitudes des transitoires de capacité produits par les impulsions de tension de hauteur $V_{1}$ et $V_{2}$ respectivement. Pour mesurer le signal DDLTS en fonction de $V_{1}-V_{2}$ nous fixons $V_{2}=1,5 \mathrm{~V}$ et faisons varier $V_{1}$; cette valeur de $V_{2}$ est choisie parce qu'elle est supérieure, pour le GaAs, au seuil de tension défini par Pons [8] lorsque $t_{\mathrm{p}}>0,2 \tau_{\mathrm{c}} ;$ d'autre part à cette valeur de $V_{2}$ l'influence sur le signal DDLTS de la recombinaison des porteurs au bord de la zone désertée est négligeable. Puisque $\left[\Delta C_{\mathrm{m}, 1}\left(t_{\mathrm{p}}\right)-\Delta C_{\mathrm{m}, 2}\left(t_{\mathrm{p}}\right)\right]$ est la différence entre deux points de la courbe $\Delta C_{\mathrm{m}}\left(t_{\mathrm{p}}\right)$ en fonction de $\Delta V$, appartenant à la région quasi-linéaire, la courbe représentant les variations de $\Delta C_{\mathrm{m}, 1}\left(t_{\mathrm{p}}\right)-\Delta C_{\mathrm{m}, 2}\left(t_{\mathrm{p}}\right)$ en fonction de $V_{1}-V_{2}$ doit aussi être quasi-linéaire et doit passer par l'origine ( $t_{\mathrm{p}}$ ayant une valeur fixée).

Nous avons mesuré l'amplitude maximale du signal DDLTS en fonction de $V_{1}-V_{2}$. La diode utilisée pour les mesures satisfaisait aux conditions de calcul de Pons [7, 8] (concentrations uniformes des donneurs $\left(N_{\mathrm{D}}\right)$ et des défauts $\left(N_{t}(x)\right)$ et $\left.N_{t}(x) \ll N_{\mathrm{D}}\right)$. On constate (Fig. 4) que les variations de $S_{\mathrm{m}}$ en fonction de $V_{1}-V_{2}$ sont quasi-linéaires dans un assez grand domaine de $\Delta V$ et qu'il n'y a pas effectivement de seuil de tension. Les mesures ont été effectuées pour trois valeurs de $t_{\mathrm{p}}$ : $1,10,100 \mu \mathrm{s}$. Pour toutes les valeurs de $\left(V_{1}-V_{2}\right)$ choisies, les trois mesures de $\Delta C_{\mathrm{m}}$ correspondant aux trois valeurs de $t_{\mathrm{p}}$ sont presque confondues. On en conclut que pour le défaut correspondant au pic 
choisi $\tau_{\mathrm{c}}$ est très inférieur à $1 \mu \mathrm{s}$. Des résultats semblables ont été obtenus sur d'autres substrats de GaAs que nous avons aussi caractérisés.

Notons que la mesure du signal DDLTS est encore satisfaisante pour une valeur $V_{1}-V_{2}=0,25 \mathrm{~V}$ (Fig. 4), bien qu'elle ne soit qu'environ dix fois supérieure à l'amplitude du signal haute fréquence du capacimètre utilisé (Boonton $72 \mathrm{BD}$ ).

2.6 Evaluation DU PROFil DE LA CONCENTRATION DES DÉFAUTS. - Le profil de la concentration d'un défaut est évalué à partir d'une mesure de $B$, à la température correspondant au maximum $S_{\mathrm{m}}$ du signal DDLTS de ce défaut $\left(B=S_{\mathrm{m}} / a_{1 \mathrm{~m}}\right.$ ou $\left.S_{\mathrm{m}} / b_{1 \mathrm{~m}}\right)$. Si l'on donne aux amplitudes $V_{1}, V_{2}$ des impulsions de tension de polarisation des valeurs voisines, $x_{1}$ et $x_{2}$ ont des valeurs proches; $\bar{N}_{t}(\bar{x})$ (valeur moyenne de $N_{t}(x)$ dans l'intervalle $\left.\left[x_{1}, x_{2}\right]\right)\left(\bar{x}=\left(x_{1}+x_{2}\right) / 2\right)$ est alors sensiblement égale à $N_{t}(x)$ et sa valeur est déduite de l'équation (12). En désignant par $C_{1}^{\prime}, W_{1}^{\prime}$ et $C_{2}^{\prime}, W_{2}^{\prime}\left(C_{1}^{\prime}=\varepsilon S / W_{1}^{\prime}, C_{2}^{\prime}=\varepsilon S / W_{2}^{\prime}\right)$ les valeurs de la capacité de la diode et de la largeur de sa charge d'espace à la fin de la première et de la deuxième impulsion de la tension de polarisation, on a :

$\bar{N}_{t}(\bar{x})=B N_{\mathrm{D}}(W)\left[C_{0}^{3}\left(\frac{1}{C_{1}^{\prime}}+\frac{1}{C_{2}^{\prime}}-\frac{2 \lambda_{1}}{\varepsilon S}\right)\left(\frac{1}{C_{2}^{\prime}}-\frac{1}{C_{1}^{\prime}}\right)\right]^{-1}$

avec

$$
\bar{x}=\frac{x_{1}+x_{2}}{2} \text { et } \lambda_{1}=W_{1}^{\prime}-x_{1}=W_{2}^{\prime}-x_{2} .
$$

Posons $\tau_{\mathrm{e}}=e_{n}^{-1}$ et $\tau_{\mathrm{c}}=\left(C_{n} N_{\mathrm{D}}(W)\right)^{-1}$. Dans le cas où $N_{t}(x) \ll N_{\mathrm{D}}(W)$ et $\tau_{\mathrm{c}} \ll t_{\mathrm{p}} \ll \tau_{\mathrm{e}}$ Pons [7, 8] a montré que :

$$
\lambda_{1}=L_{\mathrm{D}} \sqrt{2}\left[\operatorname{Ln}\left(\beta_{0} t_{0} / \tau_{\mathrm{c}}\right)-1\right]
$$

avec

$$
\beta_{0}=1 / \operatorname{Ln}\left[2-\exp \left(-e_{n}\left(\left(T_{0} / 2\right)-t_{\mathrm{p}}\right)\right]\right.
$$

(les défauts émettent des électrons pendant une durée de $T_{0} / 2-t_{\mathrm{p}}$ dans la première et dans la deuxième demi-période) et

$$
L_{\mathrm{D}}=\left(\varepsilon k T / q^{2} \bar{N}_{\mathrm{D}}^{\prime}\right)^{1 / 2}
$$

où $\bar{N}_{\mathrm{D}}^{\prime}$ est la valeur moyenne de $N_{\mathrm{D}}(W)$ dans l'intervalle $\left[x_{1}, W_{2}^{\prime}\right]$.

Comme $N_{t}(x) \ll N_{\mathrm{D}}$, les largeurs $W_{1}^{\prime}$ et $W_{2}^{\prime}$ de la charge d'espace de la diode juste avant la fin de la première et de la deuxième impulsion de tension sont pratiquement indépendantes de la charge électrique des défauts qui y sont localisés. De l'équation (13), nous déduisons également que : $x_{2}-x_{1}=W_{2}^{\prime}-W_{1}^{\prime}$ est indépendant de $t_{\mathrm{p}}$ dès que $t_{\mathrm{p}}>10 \tau_{\mathrm{c}}$ environ [8] bien que $\lambda_{1}$ varie : l'amplitude du signal DDLTS est donc indépendante de $t_{\mathrm{p}}$ lorsque $t_{\mathrm{p}}>10 \tau_{\mathrm{c}}$ puisque tous les défauts de l'intervalle constant $] x_{1}, x_{2}$ [ sont remplis d'électrons.

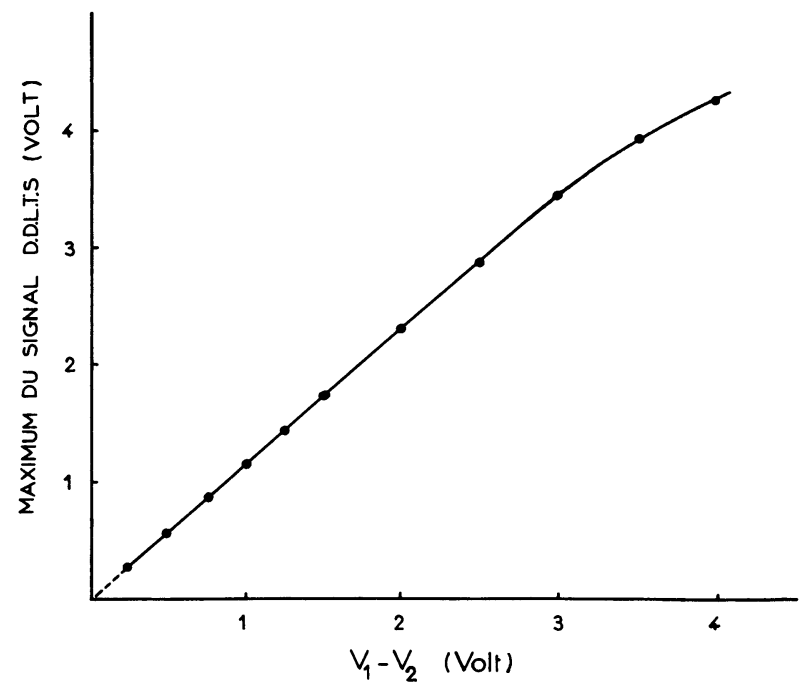

Fig. 4. - Variations du maximum du signal DDLTS $\left(a_{1}\right)$ en fonction de la différence d'amplitude entre les deux impulsions de tension de même durée $t_{\mathrm{p}}$. Les mêmes résultats ont été obtenus pour $t_{\mathrm{p}}=1,10$ et $100 \mu \mathrm{s}$. Les paramètres de mesure sont : $V_{\mathrm{B}}=-6,5 \mathrm{~V}, T=182 \mathrm{~K}, V_{2}=1,5 \mathrm{~V}$, fréquence $=31,25 \mathrm{~Hz}, e_{n}^{-1}=5,1 \times 10^{-3} \mathrm{~s}$. Substrat GaAs (100) type $\mathrm{n}\left(N_{\mathrm{D}}=1,4 \times 10^{16} \mathrm{~cm}^{-3}\right)$ recuit sous encapsulation de $\mathrm{Si}_{3} \mathrm{~N}_{4}$ à $650^{\circ} \mathrm{C}$ pendant 15 minutes.

[Plot of the maximum of DDLTS signal $\left(a_{1}\right)$ as a function of the refilling pulse $\Delta V ; \Delta V$ is the difference between two pulses having the same duration $t_{\mathrm{p}}$. Results are obtained with following parameters $V_{\mathrm{B}}=-6.5 \mathrm{~V}, T=182 \mathrm{~K}, V_{2}=$ $1.5 \mathrm{~V}, f=31.25 \mathrm{~Hz}, e_{n}^{-1}=5.1 \times 10^{-3} \mathrm{~s}, \mathrm{n}-\mathrm{GaAs}$ substrat $\left(N_{\mathrm{D}}=1.4 \times 10^{16} \mathrm{~cm}^{-3}\right)$ annealed with $\mathrm{Si}_{3} \mathrm{~N}_{4}$ encapsulant $\left(15 \mathrm{~min}\right.$ at $\left.650^{\circ} \mathrm{C}\right)$. Identical results are obtained for $t_{\mathrm{p}}=1$, $10,100 \mu \mathrm{s}$.

Pour évaluer la concentration $\bar{N}_{t}(\bar{x})$ de chaque défaut, on mesure la capacité de la diode en fonction de la tension de polarisation à la température du maximum du pic considéré dans le spectre DDLTS; par dérivation numérique de cette courbe on déduit les variations de la concentration $N_{\mathrm{D}}(W)$ en fonction de $W$. Pour évaluer $B$ on mesure le maximum de $a_{1}$ ou de $b_{1}$ en fonction de la tension de polarisation $V_{\mathbf{B}}$, les amplitudes des deux impulsions de polarisation étant gardées constantes : la concentration des défauts est donc mesurée avec une même valeur moyenne du champ électrique. Pour cette dernière mesure il faut choisir une durée $t_{\mathrm{p}}$ des impulsions de tension suffisante pour que tous les défauts de l'intervalle $] x_{1}, x_{2}[$ soient occupés par des électrons; cela est obtenu lorsque l'augmentation de la durée des impulsions de tension de $t_{\mathrm{p}}$ à $5 t_{\mathrm{p}}$ ne modifie pas la valeur du signal DDLTS.

Le profil de la concentration des défauts peut être aussi déterminé par la méthode DLTS. Johnson et al. [2] ont discuté une première solution. Une autre méthode utilisant un procédé semblable à celui de la méthode DDLTS peut également être mise en œuvre : la faible valeur de l'amplitude de l'impulsion de tension $V$ est maintenue constante $\left(\Delta V=\left|V_{0}-V_{1}\right|\right.$; 
$V_{0}$ et $V_{1}$ représentent respectivement la polarisation inverse et la polarisation pendant l'impulsion de tension) et on mesure l'amplitude maximale du signal DLTS en fonction de la tension de polarisation inverse de la diode. Dans cette méthode le signal DLTS est d'autant plus influencé par la recombinaison des porteurs en bord de zone désertée que $\Delta V$ est plus faible. Par contre, ce n'est pas le cas dans la méthode DDLTS puisque l'intervalle ] $x_{1}, x_{2}$ [ est situé en dehors de cette zone.

Nous avons représenté (Fig. 5) l'allure des fractions de défauts remplis d'électrons $\Delta f\left(x, t_{\mathrm{p}}\right)[8]$ en fonction de la profondeur à la fin d'une part de la première impulsion $(A B C D)$ et de la deuxième impulsion $\left(A^{\prime} B^{\prime} C D\right)$ de tension de DDLTS, et, d'autre part, à la fin de l'impulsion de tension de DLTS (EFCD).

Le signal DDLTS traduit l'émission des électrons par les défauts dans l'intervalle $] x_{1}, x_{2}[$. Il est proportionnel à la surface délimitée par la courbe $\mathrm{ABB}^{\prime} \mathrm{A}^{\prime}$. Les deux limites $\mathrm{AB}$ et $\mathbf{A}^{\prime} \mathbf{B}^{\prime}$ de cette surface sont identiques; elles se déduisent l'une de l'autre par une translation suivant l'axe des $x$. Cette propriété reste toujours valable même lorsque $\left(V_{1}-V_{2}\right)$ devient petit; c'est ce qui explique que le point correspondant à $V_{1}-V_{2}=0,25 \mathrm{~V}$ soit encore aligné avec les autres (Fig. 4) (pas de seuil de tension).

Le signal DLTS est, lui, proportionnel à la surface délimitée par la courbe EFCD. Les limites EF et CD

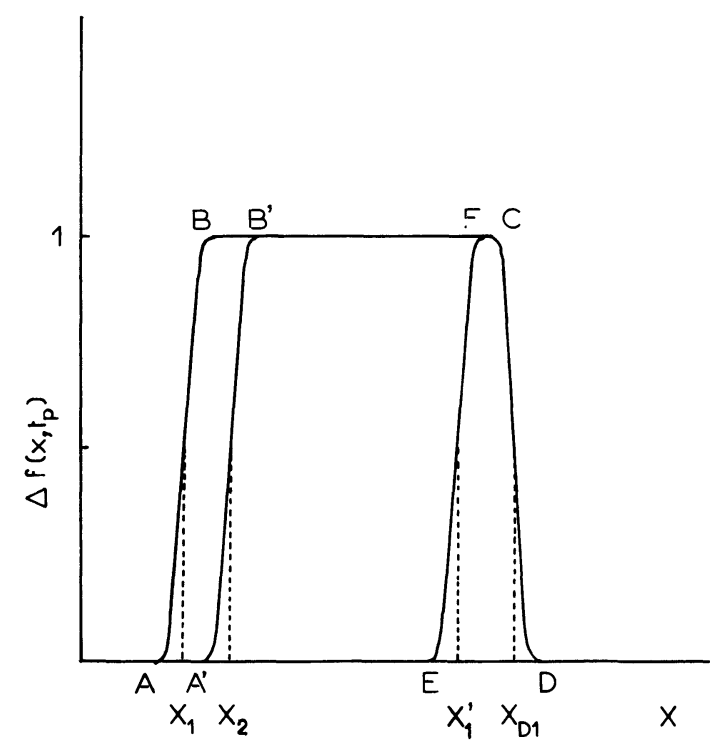

Fig. 5. - Allure des variations de la fraction de défauts remplis d'électrons $\Delta f\left(x, t_{\mathrm{p}}\right)$ en fonction de la profondeur (cas des valeurs de $t_{\mathrm{p}}>10 \tau_{\mathrm{c}}$ [8]) à la fin de la première impulsion $(A B C D)$, de la deuxième impulsion ( $\left.A^{\prime} B^{\prime} C D\right)$ dans la méthode DDLTS, et, à la fin de l'impulsion de tension (EFCD) dans la méthode DLTS.

[Fraction of the filled traps $\Delta f(x, t)$ versus the distance from the metal-semiconductor interface $\left(t_{\mathrm{p}}>10 \tau_{\mathrm{c}}\right)$ at the end respectively of the first (ABCD) and the second $\left(\mathrm{A}^{\prime} \mathrm{B}^{\prime} \mathrm{CD}\right)$ refilling pulses in DDLTS, and at the end of the refilling pulse in DLTS (EFCD).] ont des allures d'autant plus différentes que $\Delta V$ est plus petit. L'approximation de EFCD par un rectangle n'est valable que si la durée $t_{\mathrm{p}}$ est suffisamment grande pour que l'équilibre thermodynamique soit atteint.

D'autre part, d'après les courbes présentées par Pons [8] la largeur de $\Delta f\left(x, t_{\mathrm{p}}\right)$ (méthode DLTS) devient indépendante de $t_{\mathrm{p}}$ lorsque $t_{\mathrm{p}}>10 \tau_{\mathrm{e}}$ environ; en effet, au point $X_{1}^{\prime}$ où $f\left(X_{1}^{\prime}, t_{\mathrm{p}}\right)=1 / 2$ (Fig. 5) la constante d'évolution de la capacité est $e_{n}+n(x) C_{n}=$ $2 e_{n}$. Dans la méthode DDLTS le signal est produit par la- différence entre les courbes $A B C D$ et $A^{\prime} B^{\prime} C D$ (Fig. 5); ce signal devient indépendant de $t_{\mathrm{p}}$ lorsque la surface délimitée par le contour $\mathrm{ABA}^{\prime} \mathrm{B}^{\prime}$ ne varie plus avec $t_{\mathrm{p}}$ (c'est-à-dire lorsque $t_{\mathrm{p}}>10 \tau_{\mathrm{c}}$ environ [8]). Les mesures pour déterminer la concentration des défauts en fonction de la profondeur ne sont effectuées que lorsque le signal DDLTS devient indépendant de $t_{\mathrm{p}}$. Ce critère est difficile à utiliser pour la DLTS mettant en œuvre une détection synchrone, particulièrement lorsque $\Delta V$ est faible, en effet, il n'est pas toujours possible de satisfaire la condition $t_{\mathrm{p}}>10 \tau_{\mathrm{e}}$. La méthode DDLTS est donc plus appropriée pour mesurer la concentration des défauts que la méthode DLTS dans le cas où l'amplitude de l'impulsion de tension est petite.

\section{Dispositif expérimental.}

Dans le montage expérimental un interrupteur électronique placé devant le capacimètre permet d'appliquer des impulsions de tension de courte durée pour polariser la diode; il permet aussi d'éviter la saturation du capacimètre pendant la durée de l'impulsion de tension de polarisation. Le capacimètre produit un signal transitoire de durée $1,6 \mathrm{~ms}$ [6] après la fin de tension de polarisation; pour rendre négligeable ce signal transitoire, l'échantillonneur-bloqueur n'applique le signal de capacité à l'entrée de la détection synchrone qu'avec un retard de $1,6 \mathrm{~ms}$ sur la fin de l'impulsion de polarisation.

L'ensemble des appareils du montage est piloté par un microordinateur. Les impulsions de tension sont fournies soit par deux générateurs, soit par une interface digital-analogique (DAC). Cette dernière solution est intéressante par le choix des formes de la tension de polarisation qu'elle offre, mais présente l'inconvénient d'avoir un bruit de fond important provenant des signaux logiques du microordinateur. L'acquisition et l'asservissement de température sont assurés par l'intermédiaire d'une interface PIA. Les signaux de sortie de la détection synchrone sont mesurés à l'aide d'une interface analogique-digital (ADC) et sauvegardés sur disque.

\section{Résultats.}

Nous donnons ici les résultats illustrant l'application de notre méthode DDLTS à l'étude des défauts des échantillons de $\mathrm{GaAs}$ multi-implanté en oxygène. 
Ces résultats (signature et concentration des défauts) font partie d'un ensemble plus complet qui sera publié ultérieurement.

4.1 Príparation des échantillons. - Les diodes Schottky ont été réalisées sur des échantillons de GaAs (100), type $\mathrm{n}\left(1,4 \times 10^{16} \mathrm{~cm}^{-3}\right)$, multi-implantés en oxygène aux doses et énergies suivantes : $1,4 \times 10^{11} \mathrm{~cm}^{-2}, 150 \mathrm{keV} ; 1,05 \times 10^{11} \mathrm{~cm}^{-2}, 290 \mathrm{keV}$; $4,2 \times 10^{10} \mathrm{~cm}^{-2}, 550 \mathrm{keV}$. Le recuit après implantation est effectué à $650^{\circ} \mathrm{C}$ pendant $15 \mathrm{~min}$. Les contacts Schottky et ohmique ont été préparés par l'intermédiaire d'un dépôt d'or (face avant) et d'alliage Au-Ge (face arrière) respectivement.

Les doses et énergies des impuretés "oxygène" des différentes implantations ont été choisies pour obtenir leur répartition calculée sensiblement linéaire dans une région comprise entre 0,25 et $0,75 \mu \mathrm{m}$. Cette répartition linéaire qui devrait rendre plus précise la comparaison entre le profil mesuré des impuretés implantées et celui calculé, permettra de déterminer l'influence des différents paramètres (taux de dopage du substrat, température de recuit, diffusion etc...) sur la distribution des ions implantés. D'autre part, par rapport au cas d'une seule implantation, le gradient de la distribution des impuretés implantées est plus faible et la concentration électronique, déduite de la mesure de la capacité de la diode en fonction de la tension de polarisation, est alors plus proche de la concentration effective des dopants : $N_{\mathrm{D}}-N_{\mathrm{A}}\left(N_{\mathrm{A}}\right.$ est la concentration résiduelle des accepteurs).

4.2 Signature Des Défauts. - Le spectre DDLTS (Fig. 6) pour $t_{0}=T_{0} / 4$ met en évidence quatre défauts dans notre échantillon, nous notons (a), (b), (c) les trois principaux pics. Au pic (b) est superposé un pic voisin plus petit. La séparation de ces deux pics est plus nette dans le spectre de $a_{1}$ que dans celui de $b_{1}$ : la sélectivité est donc meilleure pour $a_{1}$ que pour $b_{1}$. Les maxima des pics du signal $a_{1}$ sont plus petits que ceux des pics du signal $b_{1}$; le rapport signal/bruit est donc moindre pour $a_{1}$ que pour $b_{1}$.

Les signatures des trois défauts (a), (b), (c) (Fig. 7) sont déduites des spectres de $a_{1}$ et de $b_{1}$ comme nous l'avons expliqué précédemment. Nous avons présenté sur la même figure 7 les signatures extrapolées des défauts caractérisés par G. M. Martin et al. [10] (EL3, EL7, EL9) et par Blood et al. [12] (M3), qui sont les plus proches des nôtres.

Le choix des points de mesure dans les spectres de $a_{1}$ et $b_{1}$ pour calculer les taux d'émission des électrons devient délicat lorsqu'au pic d'un premier défaut est superposé celui d'un défaut voisin (par exemple pic(b) : Fig. 6), le tracé de $\log \left(T^{2} / e_{n}\right)=f(1 / T)$ (Fig. 7) permet d'éliminer les valeurs de $T^{2} / e_{n}$ qui, en raison de cette superposition, s'écartent trop de l'alignement moyen des points obtenus.

Une régression linéaire permet d'évaluer l'énergie d'activation $E_{\mathrm{a}}$ (à partir de la pente de $\log \left(T^{2} / e_{n}\right)=$ $f(1 / T)$, l'erreur (à partir de l'écart type) et la section

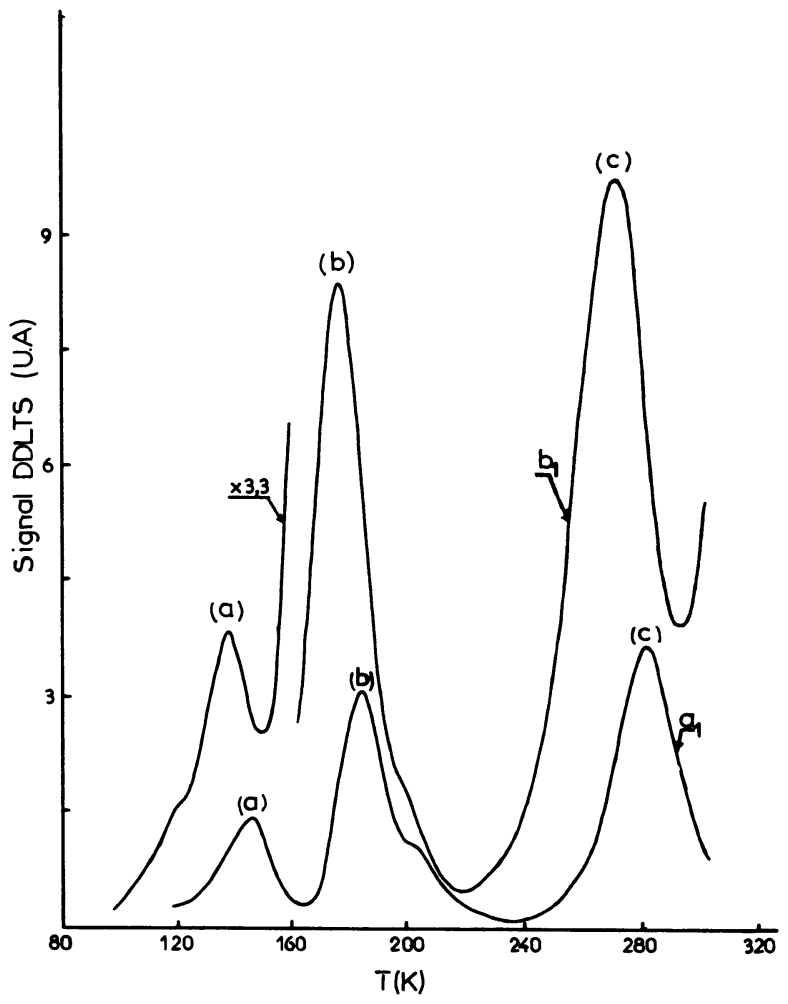

Fig. 6. - Signal DDLTS, en fonction de la température, d'un échantillon de GaAs (type $n$ ) multi-implanté en oxygène $1,4 \times 10^{11} \mathrm{~cm}^{-2}, 150 \mathrm{keV} ; 1,05 \times 10^{11} \mathrm{~cm}^{-2}, 290 \mathrm{keV}$; $4,2 \times 10^{10} \mathrm{~cm}^{-2}, 550 \mathrm{keV}$. Fréquence de mesure : $31,25 \mathrm{~Hz}$; $V_{1}=2,5 \mathrm{~V}, V_{2}=1,5 \mathrm{~V} ; V_{\mathrm{B}}=-5,5 \mathrm{~V} ; t_{\mathrm{p}}=200 \mu \mathrm{s}$.

[Variations of DDLTS signals $\left(a_{1}\right.$ and $\left.b_{1}\right)$ with temperature of oxygen multi-implanted into GaAs $\left(N_{\mathrm{D}}=1.4 \times\right.$ $10^{16} \mathrm{~cm}^{-3}$ ). Implantation parameters : $150 \mathrm{keV}, 1.4 \times$ $10^{11} \mathrm{~cm}^{-2} ; 290 \mathrm{keV}, 1.05 \times 10^{11} \mathrm{~cm}^{-2} ; 550 \mathrm{keV}, 4.2 \times$ $10^{10} \mathrm{~cm}^{-2}$. Measurement parameters : $f=31.25 \mathrm{~Hz}, V_{1}=$ $2.5 \mathrm{~V}, V_{2}=1.5 \mathrm{~V}, V_{\mathrm{B}}=-5.5 \mathrm{~V}, t_{\mathrm{p}}=200 \mu \mathrm{s}$.

efficace de capture apparente $\sigma_{\infty}$; ces grandeurs sont présentées dans le tableau IV; nous y avons ajouté les références données par G. M. Martin et al. et par Blood et al. aux défauts les plus proches de ceux que nous avons mis en évidence.

Le défaut (a) est commun à des substrats de GaAs préparés de différentes manières; selon $D$. C. Look et al. [13], ce défaut est dû à l'imperfection du réseau cristallin (défaut de réseau) plutôt qu'à une impureté.

Le défaut (b) est plus proche de EL7 [10] (Fig. 7) que de M3 [12]. Signalons que les défauts EL7 et M3 ont été mis en évidence sur des échantillons de GaAs préparés par M.B.E. à des températures voisines de celle du recuit de nos échantillons, tandis que le nôtre l'était par L.E.C.

Le défaut (c) (EL3) qui, seul, n'est pas présent dans l'échantillon de référence (non implanté et recuit dans les mêmes conditions que l'échantillon multi-implanté) est donc produit par l'implantation de l'oxygène. 
Tableau IV. - Energie d'activation $E_{\mathrm{a}}$ et section efficace apparente de capture $\sigma_{\infty}$ des défauts (a), (b), (c); signature des défauts proches des nôtres selon G. M. Martin et al. [10] et P. Blood et al. [12].

[Activation energy $E_{\mathrm{a}}$ and apparent capture cross section $\sigma_{\infty}$ of (a), (b), (c) levels. Deep levels : - after G. M. Martin et al. [10] : EL3, EL7, EL9; — after P. Blood et al. [12] : M3.]

\begin{tabular}{c|c|c|c} 
Défaut & $E_{\mathrm{a}}(\mathrm{eV})$ & $\sigma_{\infty}\left(\mathrm{cm}^{-2}\right)$ & $\begin{array}{c}\text { Signature des défauts, proches des nôtres, } \\
\text { selon G. M. Martin } \text { et al. }\left({ }^{*}\right) \\
\text { et selon P. Blood } \text { et al. }\left(^{* *}\right)\end{array}$ \\
\hline (a) & $0,2 \pm 0,02$ & $6 \times 10^{-16}$ & $\begin{array}{l}\text { EL9 }\left(^{*}\right) \\
\text { EL7 }\left(^{*}\right) \\
\text { M3 }\left(^{* *}\right) \\
\text { EL3 }\left(^{*}\right)\end{array}$ \\
(b) & $0,29 \pm 0,01$ & $5 \times 10^{-15}$ & $1,2 \times 10^{-13}$
\end{tabular}

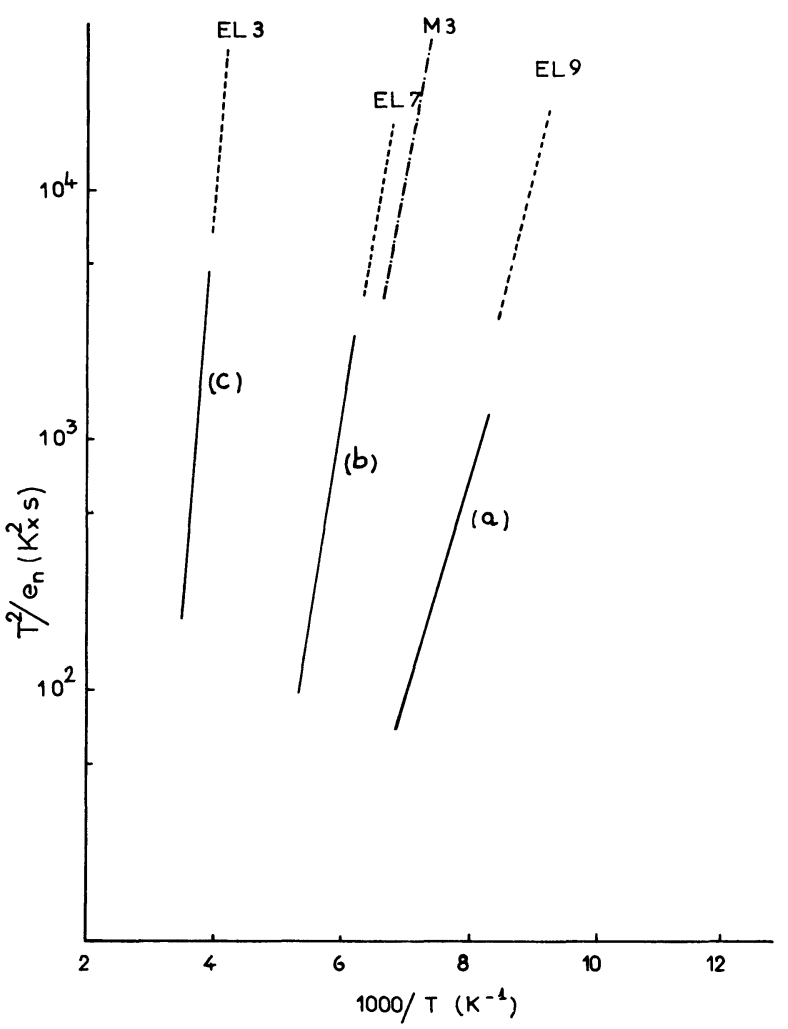

Fig. 7. - Courbes de $\log \left(T^{2} / e_{n}\right)$ en fonction de $1000 / T$. On représente les signatures des trois défauts (a), (b), (c) que nous avons mis en évidence et celles des défauts qui leur sont proches selon G. M. Martin et al. [10] (EL3, EL7, EL9) et selon P. Blood et al. [12] (M3).

[Plot of $\log \left(T^{2} / e_{n}\right)$ as a function of $10^{3} / T$; (a), (b), (c) : our results; EL3, EL7, EL9 deep levels after G. M. Martin et al. [10] and M3 after P. Blood et al. [12].]

Signalons que G. M. Martin et al. [14] ont révélé l'existence d'un phénomène analogue sur des échantillons de GaAs préparés selon la méthode de Bridgman avec adjonction d'oxygène sous forme de $\mathrm{Ga}_{2} \mathrm{O}_{3}$. Plus récemment, ces auteurs [15] ont signalé que ce centre EL3 est également présent dans les échantillons de GaAs (préparés par les méthodes Bridgman et Czochralski) après l'implantation de néon d'oxygène ou de bore. On peut donc considérer que ce défaut est un défaut du réseau cristallin résultant de l'implantation.

4.3 Profil de CONCENTRATION DES DÉfauts. - Les profils de concentration des trois défauts caractérisés précédemment ont été déterminés en maintenant constantes les amplitudes des impulsions de tension et en faisant varier la polarisation inverse de la diode $\left(V_{1}=2 \mathrm{~V} ; V_{2}=1,5 \mathrm{~V}\right)$. Pour chacun des trois défauts, la concentration est déduite de la mesure du signal DDLTS $a_{1}$, au maximum du pic correspondant. Les concentrations des défauts déterminées (Fig. 8) sont les valeurs moyennes dans l'intervalle ]$x_{1}, x_{2}[$; ces intervalles sont indiqués par des barres horizontales. Nous représentons également sur cette figure la répartition calculée de l'oxygène implanté en fonction de la profondeur. La concentration du défaut (a) est environ dix fois plus faible que celles des défauts (b) et (c). Les concentrations de (a) et (b) augmentent légèrement avec la profondeur tandis que celle du défaut (c) diminue mais varie dans le même sens que celle de l'oxygène. D'autre part les concentrations de ces trois défauts sont nettement plus faibles que celle calculée de l'oxygène.

\section{Conclusion.}

Nous avons décrit une méthode DDLTS utilisant une détection synchrone sélective; sa mise en œuvre demande un générateur d'impulsions ayant deux sorties avec réglage séparé des amplitudes et une seule détection synchrone; rappelons que la méthode basée sur la DLTS de Lang demande deux intégrateurs de signaux séparés. Par ailleurs dans notre DDLTS le signal de sortie de la détection synchrone caractérise le signal exponentiel de capacité par toutes ses valeurs pendant $T_{0} / 4$ ou $3 T_{0} / 8\left(T_{0}:\right.$ période) tandis que, dans la méthode basée sur la DLTS de Lang, ce même signal exponentiel n'est caractérisé que par deux échantillons. 


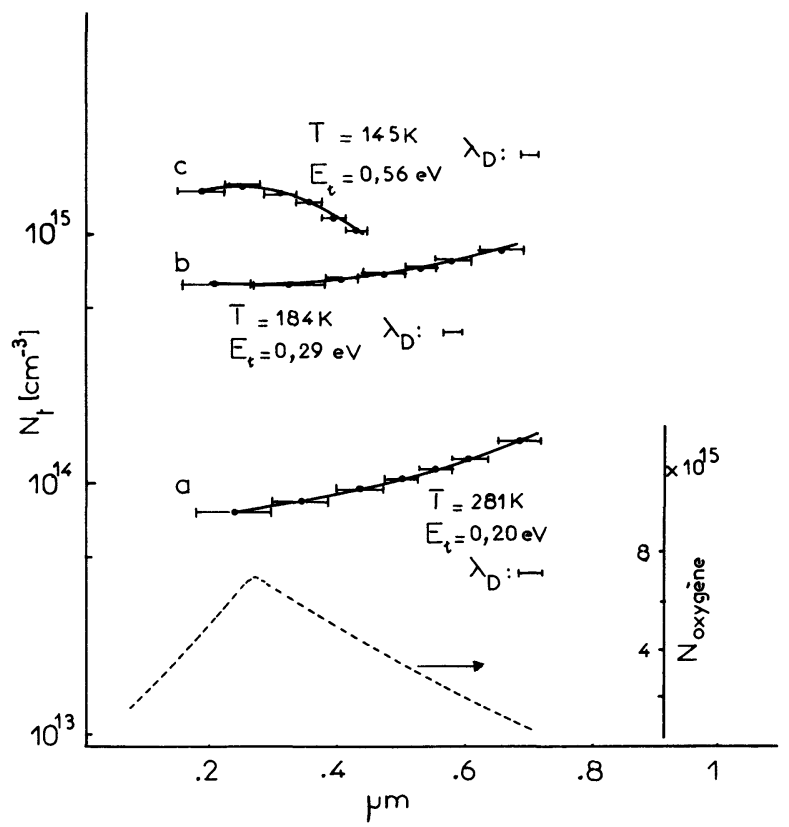

Par rapport à la DLTS, notre méthode DDLTS présente les avantages suivants :

- elle rend négligeable la contribution du signal due à la recombinaison des porteurs au bord de la zone désertée de la charge d'espace, ce qui permet une bonne détermination du profil de la concentration des défauts,

- elle détermine les taux d'émissions à champ électrique sensiblement constant.

Comme pour la méthode DDLTS basée sur la méthode DLTS de Lang [2] notre méthode peut être
Fig. 8. - Profils de concentration des trois défauts (a), (b), (c) déterminés par la méthode DDLTS. Pour chacun des profils on indique l'énergie du défaut, la température des mesures et la longueur de Debye extrinsèque $\lambda_{D}$. Les barres horizontales précisent l'intervalle $\left[x_{1}, x_{2}\right]$ sur lequel les concentrations des défauts ont été moyennées. La répartition calculée de l'oxygène implanté dans le substrat de la diode est aussi présentée (courbe en traits discontinus). Les caractéristiques de l'échantillon et les paramètres des mesures sont ceux de la figure 6 .

[Spatial depth profiles for three defect levels. With each profile is listed the trap energy $E_{t}$ the measurement temperature $T$ and the extrinsic Debye length $\lambda_{\mathrm{D}}$. The horizontal bars mark the computed intervals over which the average defect concentrations were measured. The computed oxygen implanted concentration in the diode substrat is represented (dotted line). The implantation and measurement parameters are given at the figure 6.]

mise en œuvre en maintenant constante la capacité de la diode : le signal d'entrée de l'échantillonneurbloqueur est alors la tension de polarisation de la diode; elle convient, dans ce cas, pour la caractérisation de défauts dont la concentration n'est plus négligeable par rapport à celle des donneurs.

\section{Remerciements.}

Nous remercions G. Poiblaud de la Société R.T.C., La Radiotechnique Compelec de Caen pour nous avoir fourni gracieusement le GaAs.

\section{Bibliographie}

[1] Lefevre, H., Schultz, M., Appl. Phys. 12 (1977) 45.

[2] Johnson, N. M., Bartelink, D. J., Gold, R. B., Gibbsons, J. F., J. Appl. Phys. 50 (1979) 4828.

[3] Johnson, N. M., Appl. Phys. Lett. 34 (1979) 902.

[4] Lang, D. V., J. Appl. Phys. 45 (1974) 3023.

[5] Schott, J. T., Deangelis, H. M., White, J. R., Air Force Cambridge Laboratories Report $\mathrm{n}^{\circ}$ A.F.C.R.L.-T.R. 760024 (1976).

[6] Le Bloa, A., Favennec, P. N., Revue Phys. Appl. 17 (1982) 759.

[7] Pons, D., Thèse, Université de Paris (1979).

[8] Pons, D., J. Appl. Phys. 55 (1984) 3644.

[9] Crowell, C. R., Alipanahi, S., Solid State Electron. 24 (1981) 25.
[10] Martin, G. M., Mitonneau, A., Mircea, A., Electron. Lett. 13 (1977) 893.

[11] Pons, D., Appl. Phys. Lett. 37, 4 (1980) 413.

[12] Blood, P. et Harris, J. J., J. Appl. Phys. 56 (1984) 993.

[13] LoOK, D. C., Walters, MeYer, J. R., Solid State Commun. 42 (1982) 745.

[14] Martin, G. M., Jacob, G., Hallais, J. P., Roberts, J. A., ClegG, B., Blood, P., Poiblaud, G., J. Phys. C : Solid State Phys. 15 (1982) 1841.

[15] a) Martin, G. M., Secordel, P. et Venger, C., J. Appl. Phys. 53 (1982) 8706.

b) Martin, G. M., Terriac, P., Makram-Ebeid, S., Guillot, G. et Gavan, M., Appl. Phys. Lett. 42 (1983) 61. 